\title{
Author Correction: Human genealogy reveals a selective advantage to moderate fecundity
}

Oded Galor (iD) and Marc Klemp (D)

Correction to: Nature Ecology \& Evolution https://doi.org/10.1038/s41559-019-0846-x, published online 01 April 2019.

In the version of this article initially published, several sentences contained errors. The sentence "Moreover, the analysis further suggests that evolutionary forces decreased the level of fecundity in the population over this period, consistent with an additional finding that the level of fecundity that maximized long-run reproductive success was above the population mean" contained an error. The word "above" should have been "below". Also, the sentence "Interestingly, the PI associated with the peak of the hump is above the mean and median PI in the population (Supplementary Table 3), in accordance with the finding that evolutionary forces decreased the mean PI in the population over the time period" contained an error. The word "decreased" should have been "increased". Finally, the sentence "Hence, consistent with the finding that the level of fecundity that maximized long-run reproductive success was above the population mean (that is, 62 weeks), as well as the population median (that is, 53 weeks), evolutionary forces operated towards an increase in the mean PI over these 4 generations from 62.4 to 66.2 weeks" contained an error. The word "fecundity" should have instead been "PI". The errors have been corrected in the HTML and PDF versions of the article.

Published online: 8 May 2019

https://doi.org/10.1038/s41559-019-0917-z 